\title{
Altered Biomechanical Properties of Gastrocnemius Tendons of Turkeys Infected with Turkey Arthritis Reovirus
}

\author{
Tamer A. Sharafeldin, ${ }^{1,2}$ Qingshan Chen, ${ }^{3}$ Sunil K. Mor, ${ }^{1}$ \\ Sagar M. Goyal, ${ }^{1}$ and Robert E. Porter ${ }^{1}$ \\ ${ }^{1}$ Department of Veterinary Population Medicine and Minnesota Veterinary Diagnostic Laboratory, Saint Paul, MN 55108, USA \\ ${ }^{2}$ Department of Pathology, Faculty of Veterinary Medicine, Zagazig University, Zagazig 44519, Egypt \\ ${ }^{3}$ Excelen, Center for Bone \& Joint Research and Education, Minneapolis, MN 55415, USA \\ Correspondence should be addressed to Tamer A. Sharafeldin; shara022@umn.edu
}

Received 14 March 2016; Accepted 17 November 2016

Academic Editor: Cinzia Benazzi

Copyright (C) 2016 Tamer A. Sharafeldin et al. This is an open access article distributed under the Creative Commons Attribution License, which permits unrestricted use, distribution, and reproduction in any medium, provided the original work is properly cited.

\begin{abstract}
Turkey arthritis reovirus (TARV) causes lameness and tenosynovitis in commercial turkeys and is often associated with gastrocnemius tendon rupture by the marketing age. This study was undertaken to characterize the biomechanical properties of tendons from reovirus-infected turkeys. One-week-old turkey poults were orally inoculated with O'Neil strain of TARV and observed for up to 16 weeks of age. Lameness was first observed at 8 weeks of age, which continued at 12 and 16 weeks. At 4, 8 , 12 , and 16 weeks of age, samples were collected from legs. Left intertarsal joint with adjacent gastrocnemius tendon was collected and processed for histological examination. The right gastrocnemius tendon's tensile strength and elasticity modulus were analyzed by stressing each tendon to the point of rupture. At 16 weeks of age, gastrocnemius tendons of TARV-infected turkeys showed significantly reduced $(P<0.05)$ tensile strength and modulus of elasticity as compared to those of noninfected control turkeys. Gastrocnemius tendons revealed lymphocytic tendinitis/tenosynovitis beginning at 4 weeks of age, continuing through 8 and 12 weeks, and progressing to fibrosis from 12 to 16 weeks of age. We propose that tendon fibrosis is one of the key features contributing to reduction in tensile strength and elasticity of gastrocnemius tendons in TARV-infected turkeys.
\end{abstract}

\section{Introduction}

Turkey reoviruses have often been associated with enteric diseases in turkeys [1-3]. Recently, turkey reoviruses have been isolated from gastrocnemius and digital flexor tendons and synovial fluids of 15-week-old lame turkeys, some of which had ruptured gastrocnemius or digital flexor as well as consistent histological evidence of lymphocytic tenosynovitis. These turkey arthritis reoviruses (TARVs) were found to be genetically distinct from chicken reoviruses (CARVs; [4]).

Experimental inoculation of 1-week-old turkey poults with TARV via oral, intratracheal, and footpad routes resulted in histological lesions of gastrocnemius lymphocytic tenosynovitis at 4 weeks postinoculation (PI). Such lesions were absent in poults inoculated either with turkey enteric reovirus (TERV) or with chicken arthritis reovirus (CARV) [5]. Oral inoculation of TARV in 1-week-old turkey poults induced clinical signs of lameness at 8 weeks of age, which increased in severity by 12 and 16 weeks of age [6].

Avian reoviruses that impact poultry production can result in devastating economic consequences. Avian reovirus $(\mathrm{ARV})$ is ubiquitous in domestic poultry (chickens and turkeys) with $>80 \%$ of them being nonpathogenic [7]. ARV was first isolated from birds in 1954 [8]. ARV induces tenosynovitis/arthritis in 5-7-week-old chickens resulting in lameness in heavy breeds [9]. Lameness was experimentally reproduced in chickens [10] but trials to reproduce the clinical disease in turkeys were not successful [11].

The aim of this study was to compare the biomechanical properties of gastrocnemius tendons of TARV-infected and noninfected turkeys at $4,8,12$, and 16 weeks of age. Additionally, histological alterations in the gastrocnemius tendons were described to determine if any difference in biomechanical properties could be associated with morphological 


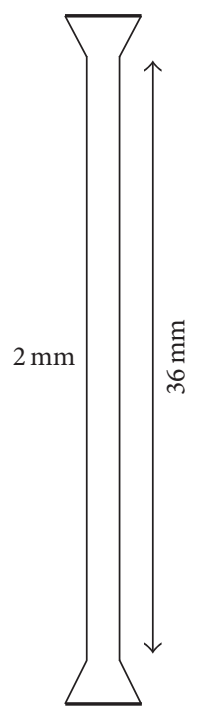

FIgURE 1: Scalloping of the outermost gastrocnemius tendon. The midsection of gastrocnemius tendon was cut as shown to create a predetermined region for measuring tendon failure.

changes in the tendons. Establishing a correlation between changes in tendon biomechanical properties with histologic and clinical findings in TARV-infected turkeys is an important aspect of TARV pathogenicity.

\section{Materials and Methods}

2.1. Samples. Samples were collected from an experimental trial [6] in which male white commercial (Nicholas) poults were divided into two groups; one group was orally inoculated at 1 week of age with $0.2 \mathrm{~mL}$ of O'Neil strain of TARV and the noninfected control group was inoculated with virus-free MEM. At 4, 8, 12, and 16 weeks of age, 10 birds from each group were euthanized followed by immediate collection of right intertarsal (hock) joint with gastrocnemius tendon, which was fixed in 10\% neutral-buffered formalin for histopathology. The skin of the left leg was removed, wrapped in gauze moistened with phosphate-buffered saline, and kept frozen $\left(-20^{\circ} \mathrm{C}\right)$ until subjected to biomechanical testing.

2.2. Histopathology. Formalin fixed intertarsal joint was decalcified in EDTA solution, trimmed, processed, and stained with hematoxylin and eosin (H\&E). Slides of stained tissues were examined under an Olympus BX40 microscope and photomicrographs were taken by Olympus DP71 digital microscope camera. Special staining was done by Masson trichrome stain for the detection of collagen, which is indicative of fibrosis.

2.3. Biomechanics. A $36 \mathrm{~mm}$ segment of the outer gastrocnemius tendon halfway between the origin (from the gastrocnemius muscle complex) and insertion (to the tarsometatarsus) was trimmed to $2 \mathrm{~mm}$ width (Figure 1) using a custommade dual blade punch. This was done to ensure that the focal point of stress in each tendon (midsection) was of

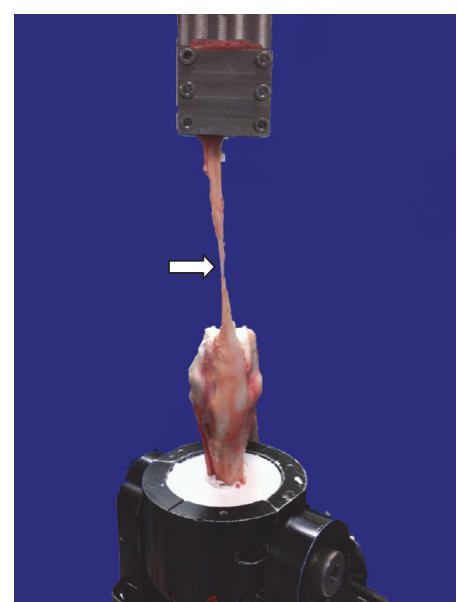

Figure 2: Orientation of leg and attached tendon and muscle. The leg of a 4-week-old turkey poult is fixed in the material testing system apparatus. Metatarsus and foot are embedded and fixed at the base of the loading frame and gastrocnemius muscle (with a portion of tendon) is clamped to the upper moveable hydraulic arm. The predetermined point of tendon failure is in the midregion of the exposed tendon (arrow).

uniform length and width. The thickness of each tendon at the trimmed midsection was determined by placing the trimmed segment between the pressure plates of a highly sensitive tabletop electromagnetic loading device (Endura TEC, ELF 3200 series, EnduraTEC Systems Corporation, Minnetonka, $\mathrm{MN})$.

The gastrocnemius tendon tensile strength and modulus of elasticity were measured by using MTS 858 Electromagnetic Material Testing System (MTS, MN, USA). The foot and tibiotarsus with attached muscle were embedded in a polyurethane potting compound (Fastcast, Goldenwest MFG, Oak Ridge, CA). The potting cylinder was then anchored to the base of the loading frame of a single pole electromagnetic device and the gastrocnemius tendon (with portion of gastrocnemius muscle) was attached to the upper hydraulic arm of the device with a fixed alligator tooth screw clamp (Figure 2). Force was exerted on each tendon to the point of stretch and complete rupture at the scalloped midsection. The unit used for measurement was megapascals $(\mathrm{MPa})$. Six tendons were analyzed for each experimental group at each time point, and measurements on tendons that did not rupture at the predetermined scalloped midsection were omitted in the final calculation. Tendon rupture in the predetermined scalloped midsection ensured that the measurements of tendon biomechanical characteristics were in the same area in different birds. The software of the device used produced a stress/strain curve for each tendon (Figure 3).

2.4. Statistical Analysis. Significant differences $(P<0.05)$ in tensile strength and elasticity of infected and noninfected groups at $4,8,12$, and 16 weeks of age were determined by using parametric and nonparametric Student's $t$-test (NCSS 8 Statistical Software, NCSS LLC, Keysville, UT). 


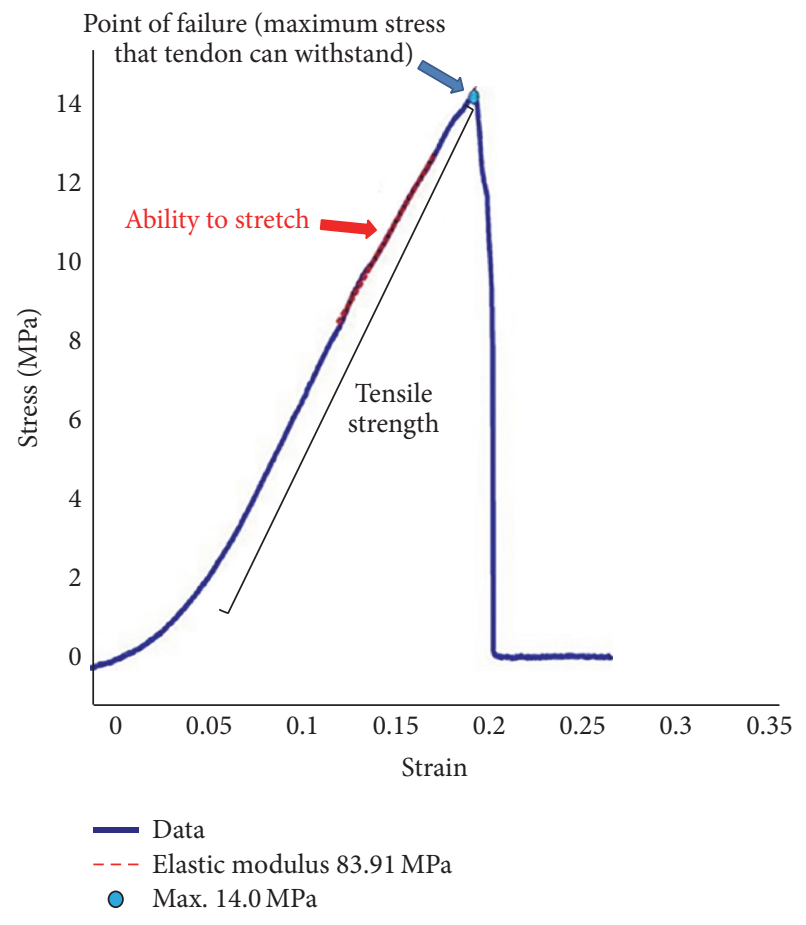

(a)

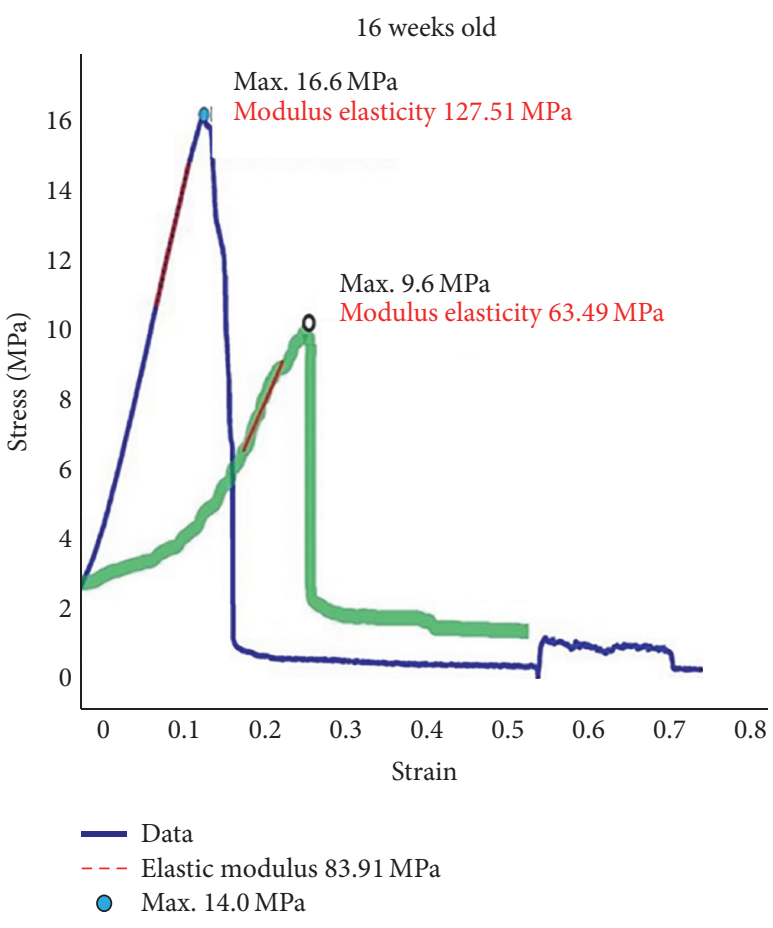

(b)

FIGURE 3: Stress/strain curves. (a) Stress/strain curve in 16-week-old turkey. (b) Stress/strain curves of median infected versus noninfected control (16 weeks of age): the blue curve with higher peak of stress refers to the median value of tensile stress in control birds and the green curve with lower peak of stress refers to the median value of tensile strength in infected birds. Unit of measurement in megapascals (MPa).

\section{Results}

3.1. Lameness and Histopathology. As shown in the previous study [6], turkeys in the infected group displayed lameness first at 8 weeks of age $(26 \%)$ and the percentage of lame birds increased at 12 and 16 weeks of age to reach $30 \%$ and $48 \%$, respectively. At 4 and 8 weeks of age, in TARVinoculated birds, the gastrocnemius tendon sheath showed prominent lymphocytic infiltration in the subsynovium along with mild synoviocyte hyperplasia. There was also mild subsynovial fibrosis in the gastrocnemius tendon of 8-weekold birds. Lymphocytic infiltration decreased at 12 weeks with increased subsynovial fibrosis which also involved the peritendon and was more prominent in 16-week-old turkeys. Masson trichrome stain revealed a progressive increase in the deposition of collagen in the subsynovium and, in some instances, on the peritendon surface of the gastrocnemius tendon and tendon sheath of 12- and 16-week-old infected turkeys (Figure 4). The tendons histologic inflammation scores were significantly higher in infected birds compared with the noninfected control at all ages [6]. Birds did not display diarrhea. However, body weights of infected birds were significantly lower than of control birds at ages of 12 and 16 weeks [6]. Birds that showed severe lameness, ruptured tendons (only 1 bird at 16 weeks of age), and/or inability to stand were euthanized.

3.2. Biomechanics. Though we had a total of 80 legs, we randomly processed only 48 for biomechanical study due to funding and time constraints. Of the 48 legs examined, we further eliminated 5 gastrocnemius tendons/group because the tendons did not rupture at the predetermined midsection of the tendons. In all instances, the aberrant ruptures occurred at the end or clamped region of the tendon. There were no significant differences between the mean tensile strength and modulus of elasticity of gastrocnemius tendons of infected and noninfected groups at 4, 8, and 12 weeks of age; however, by 16 weeks of age, the gastrocnemius tendons of infected turkeys had a significantly lower $(P<0.05)$ mean tensile strength and modulus of elasticity compared to tendons of noninfected turkeys (Table 1). Interestingly, correlation coefficient between clinical lameness and biomechanical properties (tensile strength and elasticity modulus) showed increased inverse relationship at 16 weeks of age (Figure 5).

\section{Discussion}

Clinical cases of tendon rupture associated with reovirus infection are generally observed in male turkeys older than 12 weeks of age (David Mills, personal communication). We have found that inoculation of 1-week-old turkey poults with TARV induces histologic tenosynovitis at 4 weeks PI without clinical lameness [5]. However, clinical lameness can be seen at 8 weeks PI, which later progresses at 12 and 16 weeks PI [6]. The aim of the present study was to determine the effect of TARV infection on gastrocnemius tendon tensile strength and modulus of elasticity (resistance to stretch) in turkeys. 

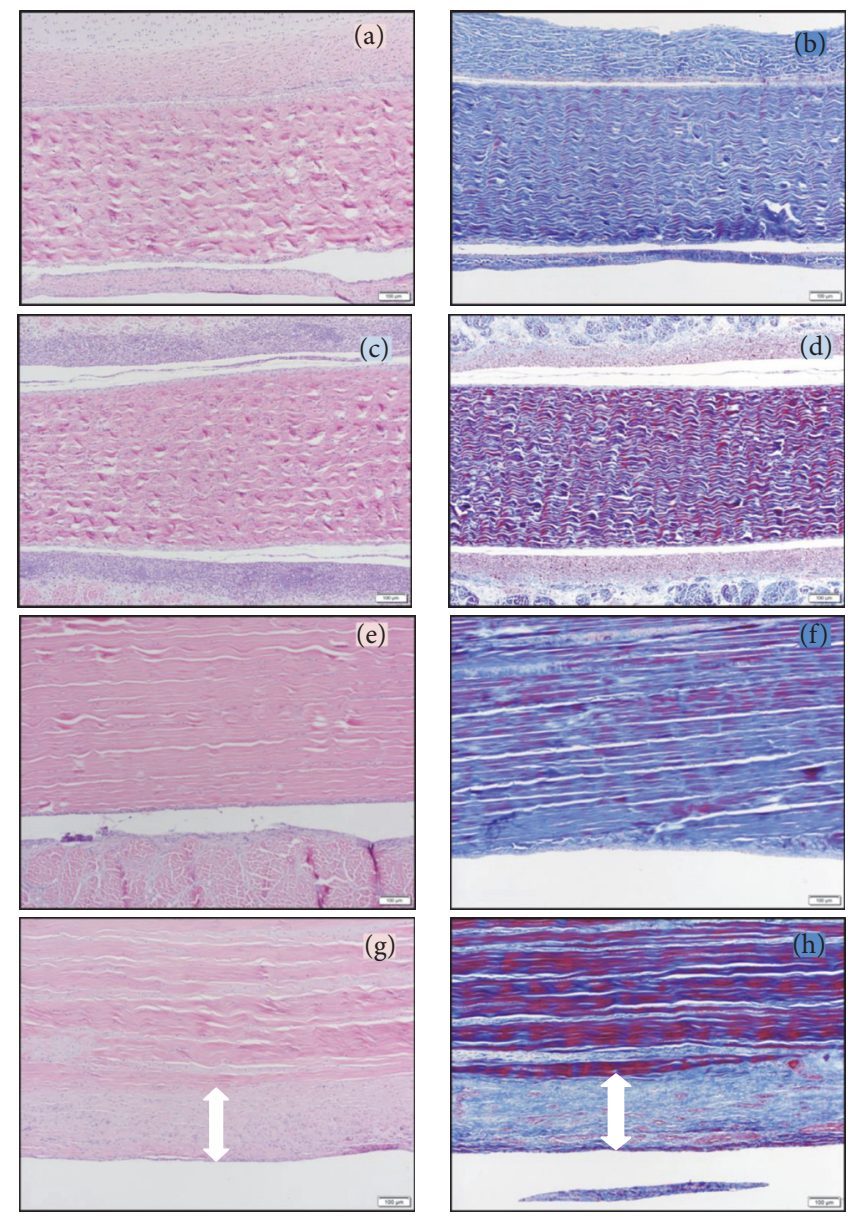

FIGURE 4: Histologic lesions in turkey poults after staining with Masson trichrome (MT) stain: (a) 4-week-old control turkey H\&E; (b) 4week-old control MT; (c) 4-week-old infected H\&E; (d) 4-week-old infected MT; (e) 16-week-old control H\&E; (f) 16-week-old control MT; (g) 16-week-old infected H\&E; (h) 16-week-old infected MT. Inflammatory cells (lymphocytes) in subsynovium in (c) and (d). Collagen (indicative for fibrosis) deposition in the tendon (blue fibrils) in (g) and (h) (double headed white arrows).

TABLE 1: Average tendon tensile strength and elasticity of turkey gastrocnemius tendons at different time points.

\begin{tabular}{lcccc}
\hline Age (weeks) & Group & Number & $\begin{array}{c}\text { Tensile strength (MPa) } \\
\text { Mean } \pm \text { SD }\end{array}$ & $\begin{array}{c}\text { Modulus elasticity (MPa) } \\
\text { Mean } \pm \text { SD }\end{array}$ \\
\hline \multirow{2}{*}{4} & Control & 6 & $15.4 \pm 4.86$ & $75.86+29.37$ \\
& Infected & 6 & $12.6 \pm 11.01$ & $67.10+41.51$ \\
8 & Control & $4^{+}$ & $9.03^{\mathrm{a}} \pm 2.12$ & $41.45+16.54$ \\
12 & Infected & 6 & $12.78 \pm 6.70$ & $51.86+22.60$ \\
& Control & 5 & $11.76 \pm 4.55$ & $57.34+2.12$ \\
& Infected & 6 & $9.03 \pm 5.56$ & $50.76+23.08$ \\
& Control & 5 & $19.36 \pm 6.59$ & $150.40+59.34$ \\
\hline
\end{tabular}

${ }^{*}$ Significantly lower than control mean at the same age at $P<0.05$, Mann-Whitney $U$ test.

${ }^{\text {a }}$ Significantly lower than control means of other ages at $P<0.05$, Mann-Whitney $U$ test.

${ }^{+} n=6$ per group. Tendon measurements were omitted if the tendon did not rupture at the predetermined midsection. SD: standard deviation.

MPa: megapascals. 


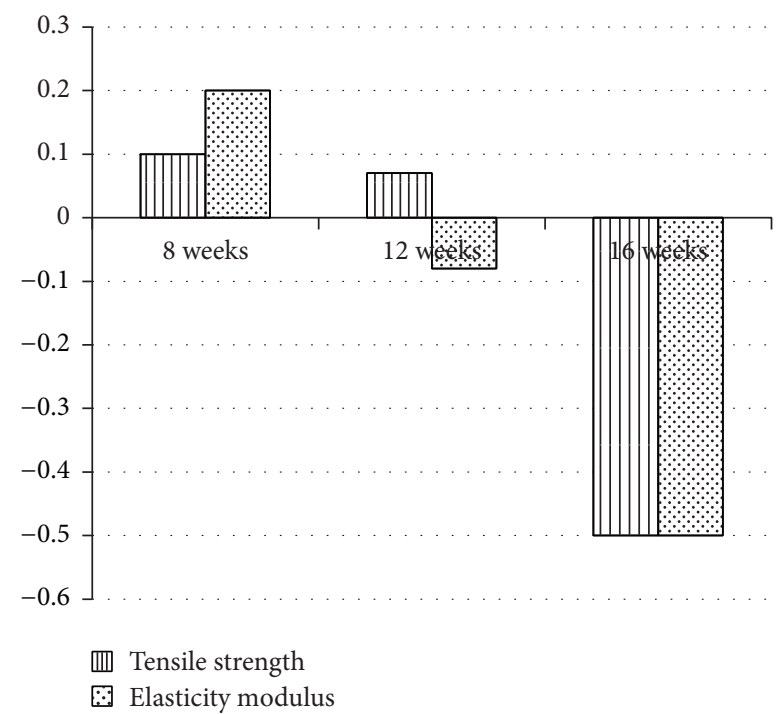

FIGURE 5: Correlation coefficient between lameness and biomechanical properties. Elevated inverse correlation between lameness and tensile strength and elasticity modulus at 16 weeks of age.

Subsynovial fibrosis first developed in 8-week-old turkeys and became predominant by 16 weeks of age. Mean tendon tensile strength and modulus of elasticity were significantly lower $(P<0.05)$ in 16-week-old infected turkeys compared to noninfected turkeys. Mean tendon tensile strength in control turkeys was significantly lower at 8 weeks of age compared with controls of all other time points (Table 1). Perhaps the reduced tensile strength of tendon in 8-week-old control turkeys is spuriously low because only 4 tendons from that group were analyzed as a result of aberrant rupture in two tendons, which were omitted from the study. The means of tendon tensile strength were the same in infected turkeys at all time points $(P<0.05)$. Mean tendon elasticity in control turkeys was the same at 4,8 , and 12 weeks while elasticity was significantly increased in noninfected 16-week-old turkeys $(P<0.05)$ (Table 1). These observations might explain the role of fibrosis in decreasing the tensile strength and modulus of elasticity in infected turkeys.

Previous studies on collagen composition and arrangement in turkey tendons have demonstrated progressive changes in procollagen content and organization as the turkey grows [12]. In our study, induction of substantial inflammation and edema in the gastrocnemius subsynovium and tendon, as well as reovirus replication in these tissues [6], may have altered physiological collagen deposition, ultimately altering tendon tensile strength and elasticity.

A study on gastrocnemius tendon biomechanics in reovirus-infected chickens showed that tendons of chickens infected at one day of age had significantly lower mean tensile strength compared with noninfected chickens at 6, 10, and 18 weeks of age $(P<0.05)$. This work also reported a gradual increase of the mean tendon tensile strength of control birds which was significantly higher only at 18 weeks compared to other time points. Additionally, the mean modulus of elasticity in controls was significantly lower than that of infected birds only at 18 weeks [13]. Our findings closely parallel those of the chicken study and can improve our understanding of the pathogenesis of TARV [14].

Changes in the biomechanical properties of tendon in the turkeys in our study may be a result of decreased movement of infected lame birds. A previous work showed that immobilized chickens had 10-30\% decreased tendon strength and $70 \%$ decreased tendon elasticity [15]. These findings were attributed to the decreased organization and diameter of collagen fibers as characterized by electron microscopy.

Finally, the progressive deposition of fibrous connective tissue on and around the tendon of reovirus-infected turkeys as they age combined with the progressive increase in body weight [6] is likely a predisposing factor in inducing lameness and, in some instances, tendon rupture. Increased inverse correlation between clinical lameness and tensile strength and elasticity modulus suggests the possible role of the decreased biomechanical properties values in increasing the clinical lameness at 16 weeks of age. In chickens, it was proposed that fibrosis and adhesions with increased age in heavyweight birds were responsible for gastrocnemius tendon and digital flexor tendon rupture [16].

In conclusion, TARV-infected turkeys developed gastrocnemius lymphocytic tenosynovitis by 4 weeks of age, progressing to fibrosis by 16 weeks of age. Clinical lameness was observed at 8 weeks and was observed in $50 \%$ of infected birds at 16 weeks. This lameness was accompanied with tendon rupture in a small percentage of 16-week-old turkeys. The progressive lameness and occasional tendon rupture represent altered tendon function (reduced tensile strength and reduced modulus of elasticity) during infection in a rapidly growing turkey.

\section{Competing Interests}

The authors declare that they have no competing interests.

\section{Acknowledgments}

The authors thank Dr. Jack Rosenberger for providing TARVO'Neil for the study. This work was supported in part by the University of Minnesota Rapid Agricultural Research Fund.

\section{References}

[1] N. Jindal, D. P. Patnayak, A. F. Ziegler, A. Lago, and S. M. Goyal, "A retrospective study on poult enteritis syndrome in Minnesota," Avian Diseases, vol. 53, no. 2, pp. 268-275, 2009.

[2] I. Lojkić, M. Biđin, Z. Biđin, and M. Mikec, "Viral agents associated with poult enteritis in Croatian commercial Turkey flocks," Acta Veterinaria Brno, vol. 79, no. 1, pp. 91-98, 2010.

[3] S. K. Mor, T. A. Sharafeldin, M. Abin et al., "The occurrence of enteric viruses in Light Turkey Syndrome," Avian Pathology, vol. 42, no. 5, pp. 497-501, 2013.

[4] S. K. Mor, T. A. Sharafeldin, R. E. Porter, A. Ziegler, D. P. Patnayak, and S. M. Goyal, "Isolation and characterization of a Turkey arthritis reovirus," Avian Diseases, vol. 57, no. 1, pp. 97103, 2013. 
[5] T. A. Sharafeldin, S. K. Mor, A. Z. Bekele, H. Verma, S. M. Goyal, and R. E. Porter, "The role of avian reoviruses in turkey tenosynovitis/arthritis," Avian Pathology, vol. 43, no. 4, pp. 371378, 2014.

[6] T. A. Sharafeldin, S. K. Mor, A. Z. Bekele et al., "Experimentally induced lameness in turkeys inoculated with a newly emergent turkey reovirus," Veterinary Research, vol. 46, article no. 11, 2015.

[7] R. C. Jones, "Reovirus infections," in Diseases of Poultry, Y. M. Saif, H. J. Barnes, J. R. Glisson, A. M. Fadly, L. R. McDougald, and D. E. Swayne, Eds., pp. 309-328, Iowa State University Press, Ames, Iowa, USA, 12th edition, 2008.

[8] J. E. Fahey and J. F. Crawley, "Studies on chronic respiratory disease of chickens II. Isolation of a virus," Canadian Journal of Comparative Medicine, vol. 18, no. 1, pp. 13-21, 1954.

[9] G. E. Wilcox, M. D. Robertson, and A. D. Lines, "Adaptation and characteristics of replication of a strain of avian reovirus in vero cells," Avian Pathology, vol. 14, no. 3, pp. 321-328, 1985.

[10] K. M. Kerr and N. O. Olson, "Pathology of chickens experimentally inoculated or contact-infected with an arthritis-producing virus," Avian Diseases, vol. 13, no. 4, pp. 729-745, 1969.

[11] A. A. Afaleq and R. C. Jones, "Pathogenicity of three turkey and three chicken reoviruses for poults and chicks with particular reference to arthritis/tenosynovitis," Avian Pathology, vol. 18, no. 3, pp. 433-440, 1989.

[12] L. Knott, J. F. Tarlton, and A. J. Bailey, "Chemistry of collagen cross-linking: biochemical changes in collagen during the partial mineralization of turkey leg tendon," Biochemical Journal, vol. 322, no. 2, pp. 535-542, 1997.

[13] F. M. Mohamed, T. L. Foutz, G. N. Rowland, and P. Villegas, "Biomechanical properties of the gastrocnemius tendon in broilers experimentally infected with avian reovirus," Transactions of the American Society of Agricultural Engineers, vol. 38, no. 6, pp. 1893-1899, 1995.

[14] T. A. Sharaf Eldin, Turkey arthritis reovirus: pathogenesis and immune response [Ph.D. thesis], University of Minnesota, 2015.

[15] T. L. Foutz, A. K. Griffin, J. T. Halper, and G. N. Rowland, "Effects of activity on avian gastrocnemius tendon," Poultry Science, vol. 86, no. 2, pp. 211-218, 2007.

[16] R. C. Jones, "Avian reovirus infections," Revue Scientifique et Technique de l'OIE, vol. 19, no. 2, pp. 614-625, 2000. 

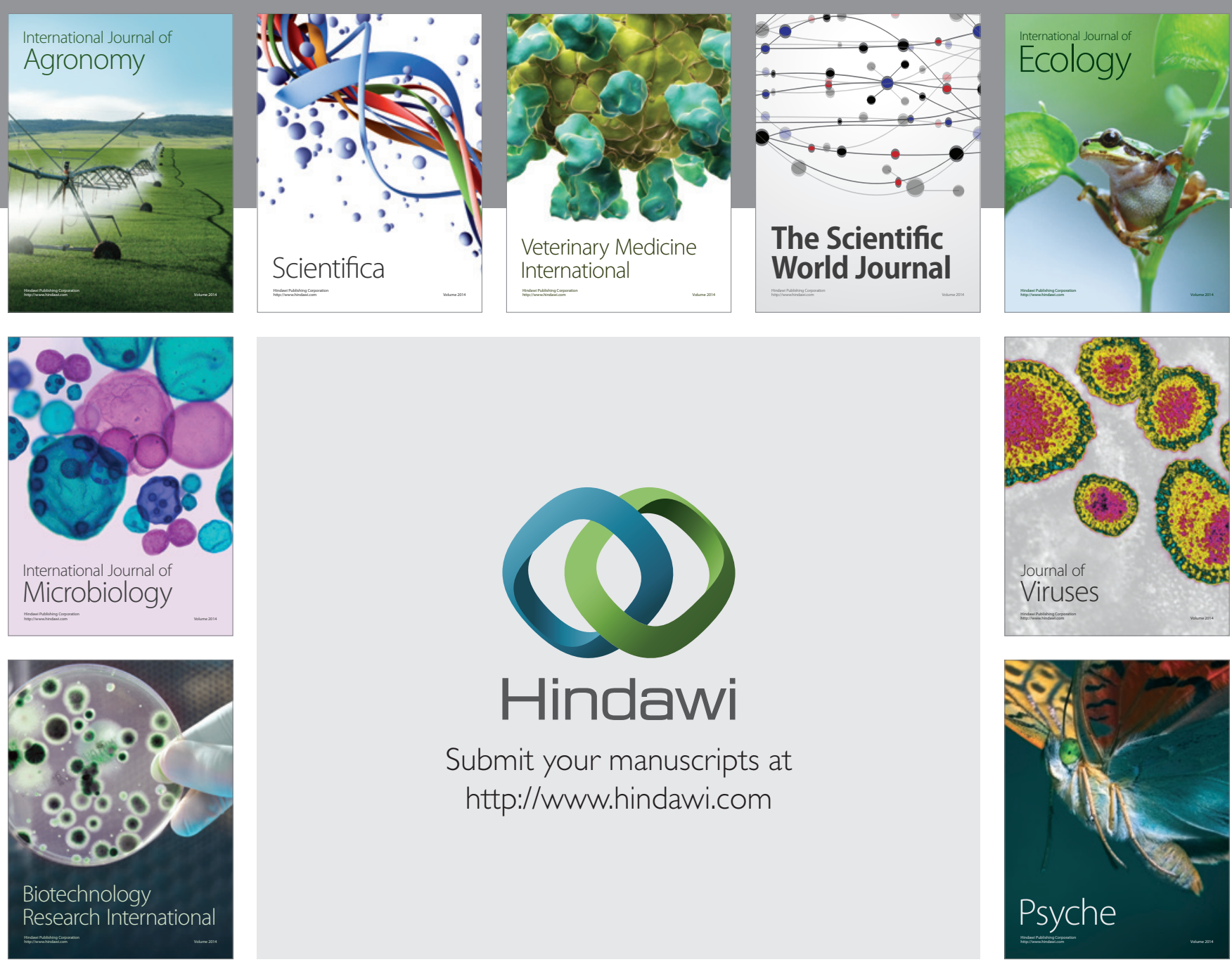

Submit your manuscripts at

http://www.hindawi.com
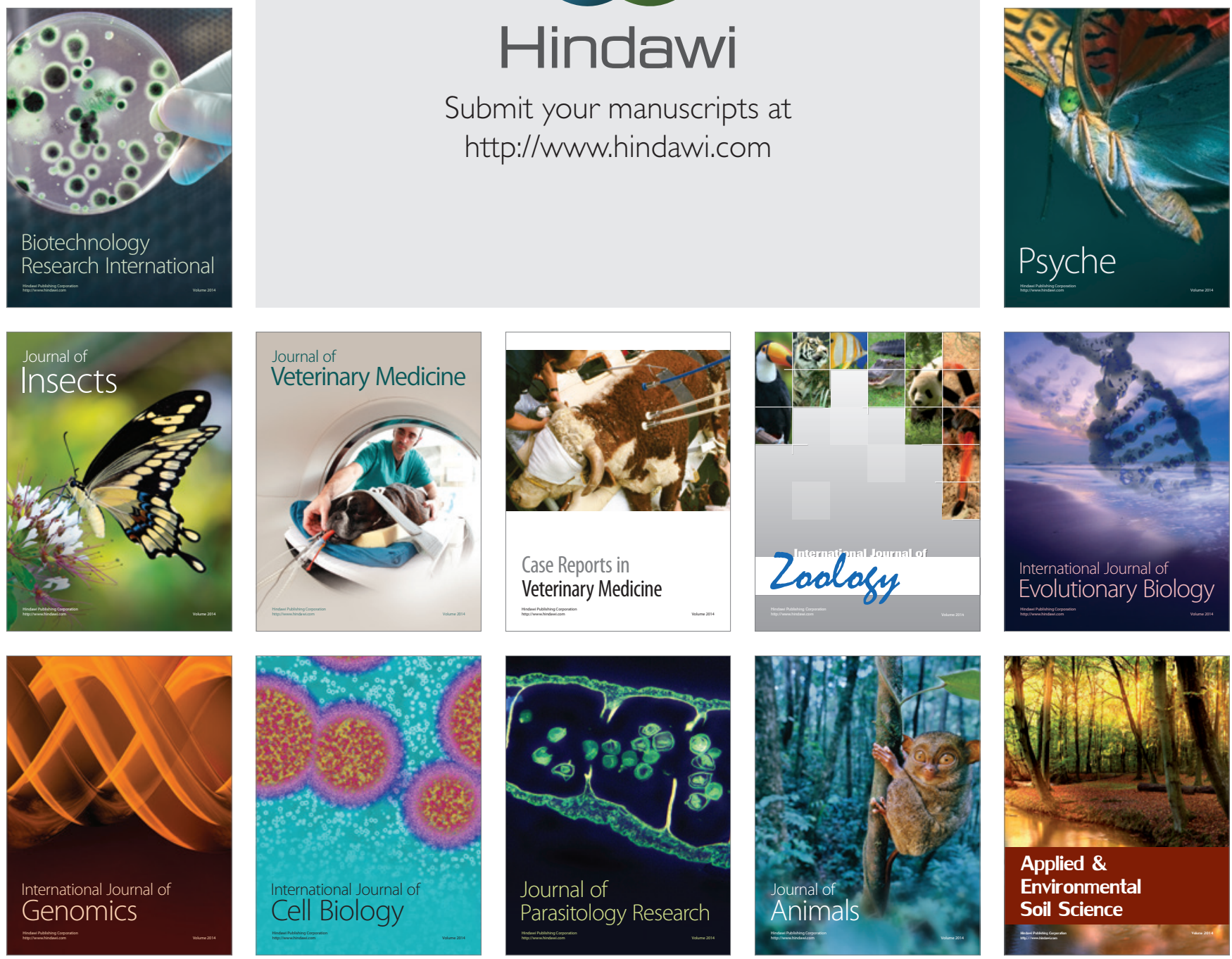